\title{
Epigenetic changes in blood leukocytes following an omega-3 fatty acid supplementation
}

\author{
Bénédicte L. Tremblay ${ }^{1}$, Frédéric Guénard ${ }^{1}$, Iwona Rudkowska², Simone Lemieux ${ }^{1}$, Patrick Couture ${ }^{1,2}$ \\ and Marie-Claude Vohl $^{1,2^{*}}$
}

\begin{abstract}
Background: Omega-3 polyunsaturated fatty acids ( $n-3$ FAs) have several beneficial effects on cardiovascular (CV) disease risk factors. These effects on CV risk profile may be mediated by several factors, including epigenetic modifications. Our objective is to investigate, using genome-wide DNA methylation analyses, methylation changes following an n-3 FA supplementation in overweight and obese subjects and to identify specific biological pathways potentially altered by the supplementation.

Results: Blood leukocytes genome-wide DNA methylation profiles of 36 overweight and obese subjects before and after a 6-week supplementation with $3 \mathrm{~g}$ of n-3 FAs were compared using GenomeStudio software. After supplementation, $308 \mathrm{CpG}$ sites, assigned to 231 genes, were differentially methylated (FDR-corrected Diffscore $\geq|13| \sim P \leq 0.05)$. Using Ingenuity Pathway Analysis system, a total of 55 pathways were significantly overrepresented following supplementation. Among these pathways, 16 were related to inflammatory and immune response, lipid metabolism, type 2 diabetes, and cardiovascular signaling. Changes in methylation levels of CpG sites within AKT3, ATF1, HDAC4, and IGFBP5 were correlated with changes in plasma triglyceride and glucose levels as well as with changes in the ratio of total cholesterol/HDL-cholesterol following the supplementation.
\end{abstract}

Conclusions: These data provide key differences in blood leukocytes DNA methylation profiles of subjects following an n-3 FA supplementation, which brings new, potential insights on metabolic pathways underlying the effects of n-3 FAs on CV health.

Keywords: DNA methylation, Omega-3 fatty acids, Microarray, Metabolic pathways, Blood leukocytes

\section{Background}

Fish-oil-derived long-chain omega-3 fatty acids (n-3 FAs), including eicosapentaenoic acid (EPA, 20:5 n-3) and docosahexaenoic acid (DHA, 22:6 n-3) have several benefits on cardiovascular (CV) health. They exert hypotriglyceridemic $[1,2]$, anti-inflammatory [3-5], antiarrhythmic [6, 7], and anti-thrombotic effects [8, 9]. Many factors, including genetic and epigenetic factors, may contribute to the observed effects of n-3 FAs on the $\mathrm{CV}$ risk profile. Indeed, emerging evidence suggests that n-3 FAs might influence global DNA methylation

\footnotetext{
* Correspondence: marie-claude.vohl@fsaa.ulaval.ca

${ }^{1}$ Institute of Nutrition and Functional Foods (INAF), Laval University, 2440 Hochelaga Blvd, Quebec, QC G1V 0A6, Canada

${ }^{2} \mathrm{CHU}$ de Québec Research Center - Endocrinology and Nephrology, 2705 Laurier Blvd, Quebec, QC G1V 4G2, Canada
}

patterns due to their role in one-carbon metabolism [10]. A study in rats fed on a vitamin $B_{12}$-deficient diet demonstrated that DHA modify DNA methylation, indicating that it plays a role in one-carbon metabolism [11]. DNA methylation is the best-characterized epigenetic factor and consists of the methylation of cytosine residues, mainly at cytosine-phosphate-guanine (CpG) dinucleotides [12]. The modification of DNA methylation by the environment may influence the regulation of $\mathrm{CV}$ risk factors, such as hypertension [13], atherosclerosis [14, 15], and inflammation [16]. Moreover, the methylation of repetitive sequences in blood has also been associated with CV diseases in epidemiological studies [17, 18].

Only a few studies have dealt with the impact of n-3 FAs on DNA methylation in human subjects. These 
studies were conducted in various populations including children and adolescents $[19,20]$, adults with renal impairment [21], women under caloric restriction [22], and Yup'ik Alaska Native individuals [23]. Moreover, a recent study demonstrated that a DHA supplementation during pregnancy was associated with changes in global methylation levels of inflammatory mediated genes [24].

The aim of this study was to investigate DNA methylation changes following n-3 FA supplementation in overweight and obese subjects and identify biological pathways potentially altered by the n-3 FA supplementation, by using whole-genome DNA methylation analyses. To our knowledge, this is the first study to examine the possible effect of n-3 FA supplementation on genomewide DNA methylation levels in blood leukocytes of overweight and obese adults.

\section{Results}

\section{Effects of $n-3$ FA supplementation}

Biochemical parameters of study subjects $(n=36)$ before and after the n-3 FA supplementation are presented in Table 1. The supplementation was associated with a decrease in fasting plasma triglyceride (TG) concentrations, similar to results reported in full cohort [25]. In the same manner, total cholesterol (TC) and the ratio $\mathrm{TC} /$ high-density lipoprotein cholesterol (HDL-C) decreased whereas glucose concentrations slightly increased after the supplementation (Table 1). As expected, the supplementation was associated with a decrease in percentage and absolute values of linoleic, arachidonic, and total n-6 FAs (including all cis and trans n-6 FAs) in plasma phospholipids ( $P$ value $<0.0001$ for all, data not shown). It was also

Table 1 Biochemical parameters of subjects before and after n-3 FA supplementation $(n=36)$

\begin{tabular}{llll}
\hline & Before $\mathrm{n}-3$ suppl. & After $\mathrm{n}$-3 suppl. & $P$ value \\
\hline Gender & 18 men and 18 women & \\
Age (years) & $34.7 \pm 8.8$ & & \\
BMI $\left(\mathrm{kg} / \mathrm{m}^{2}\right)$ & $29.2 \pm 3.65$ & $29.2 \pm 3.83$ & 0.24 \\
Triglycerides ${ }^{\mathrm{a}}(\mathrm{mmol} / \mathrm{L})$ & $1.42 \pm 0.80$ & $1.24 \pm 0.65$ & 0.0034 \\
Cholesterol (mmol/L) & & & \\
$\quad$ Total & $5.24 \pm 0.9$ & $5.12 \pm 0.92$ & 0.048 \\
$\quad$ LDL-C & $3.18 \pm 0.91$ & $3.10 \pm 0.92$ & 0.19 \\
$\quad$ HDL-C & $1.40 \pm 0.35$ & $1.44 \pm 0.40$ & 0.061 \\
Ratio TC/HDL-C & $4.0 \pm 1.31$ & $3.85 \pm 1.35$ & 0.018 \\
Glucose $(\mathrm{mmol} / \mathrm{L})$ & $4.86 \pm 0.51$ & $5.06 \pm 0.47$ & 0.0085 \\
Insulin ${ }^{\mathrm{a}}(\mathrm{pmol} / \mathrm{L})$ & $108.0 \pm 143.5$ & $91.7 \pm 48.4$ & 0.70 \\
CRP $^{\mathrm{a}}(\mathrm{mg} / \mathrm{L})$ & $3.89 \pm 6.57$ & $3.24 \pm 5.32$ & 0.18 \\
\hline
\end{tabular}

Data are shown as mean \pm SD

Abbreviations: BMI body mass index, CRP C-reactive protein, $H D L-C$ high-

density lipoprotein cholesterol, $L D L-C$ low-density lipoprotein cholesterol, suppl supplementation, $T C$ total cholesterol

${ }^{\mathrm{a}} P$ value derived from $\log _{10}$ transformed data associated with an increase in percentage and absolute values of EPA, DHA, ratio n-3/n-6, and total n-3 FAs (including all cis and trans n-3 FAs) in plasma phospholipids ( $P$ value $<0.0001$ for all, data not shown).

\section{Genome-wide DNA methylation analyses}

Globally, 484,027 of the 485,577 probes (99.7\%) on the array were detected with a detection $P$ value $\leq 0.05$. After n-3 FA supplementation, 308 CpG sites, assigned to 231 genes, were differentially methylated (false discovery rate (FDR)-corrected DiffScore $\geq|13| \sim P \leq 0.05)$. A total of 286 CpG sites were hypermethylated (93\%) and 22 were hypomethylated (7\%) after supplementation as compared to levels before supplementation (Table 2). A total of $36.4 \%$ of significant differentially methylated CpG sites were located in gene bodies (Table 2). The genomic localization of $\mathrm{CpG}$ sites is summarized in Table 2. Detailed information about the 308 differentially methylated CpG sites is presented in Additional file 1.

\section{Relationship between CpG sites and surrounding SNPs}

Using results from a recent GWAS done by our group in the same cohort [26], we tested potential relationship between pre-supplementation methylation levels and changes in methylation levels ( $\Delta$ methylation) of the 308 differentially methylated $\mathrm{CpG}$ sites and surrounding single-nucleotide polymorphisms (SNPs) $( \pm 1 \mathrm{~kb})$. Single-

Table 2 Summary of methylation results

\begin{tabular}{|c|c|}
\hline & $n$ \\
\hline Probes & 485577 \\
\hline Number of probes detected $(P \leq 0.05)$ & 484027 \\
\hline Differentially methylated (FDR-corrected DiffScore $\geq|13|$ ) & 308 \\
\hline Hypermethylated after n-3 FA supplementation & 286 \\
\hline Gene body & 107 \\
\hline 3'-UTR & 34 \\
\hline 1st exon & 4 \\
\hline $5^{\prime}-U T R$ & 19 \\
\hline Promoter region $^{a}$ & 50 \\
\hline Intergenic region & 72 \\
\hline Hypomethylated after n-3 FA supplementation & 22 \\
\hline Gene body & 5 \\
\hline $3^{\prime}-U T R$ & 0 \\
\hline 1st exon & 1 \\
\hline $5^{\prime}-U T R$ & 5 \\
\hline Promoter region ${ }^{a}$ & 6 \\
\hline Intergenic region & 5 \\
\hline
\end{tabular}

Localization according to the first annotated transcript for each CpG site and provided for the Infinium HumanMethylation450 BeadChip

Abbreviations: FDR false discovery rate, UTR untranslated region, TSS transcription start site

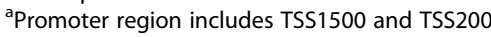


nucleotide polymorphisms (SNPs) had an effect on presupplementation methylation levels of $15 \mathrm{CpG}$ sites. Moreover, SNPs only affected $\Delta$ methylation of two CpG sites. Indeed, rs41286653 and rs899388 affected $\Delta$ methylation of cg02296904 located in the body of the gene GAK (chromosome 4). The SNP rs114329043 also affected $\Delta$ methylation of cg27270362 located in an intergenic region on chromosome 10 . We can conclude that the potential effect of the n-3 FA supplementation on $\Delta$ methylation of the $308 \mathrm{CpG}$ sites is due in very small proportion to surrounding SNPs.

\section{Pathway analyses}

From the 231 differentially methylated genes following supplementation, Ingenuity Pathway Analysis (IPA) mapped 227 genes. IPA revealed 55 pathways that were significantly overrepresented $(P \leq 0.05)$ (detailed pathways are presented in the Additional file 2), but we focused on the 16 pathways related to $\mathrm{CV}$ health. Selected $\mathrm{CV}$ health-related pathways, associated $P$ values, and differentially methylated genes identified in pathways are presented in Table 3. These selected pathways revealed relevant genes known to be related to inflammatory and immune response (AKT3, ATF1, BAX, CASP6, DHRS9, FAS, PRKAG2, PRKCZ, PRKD3, PTEN, TRIM24), lipid metabolism (AKT3, IGFBP5, KLK6, NUDT3, PLCH1, PPP2RE5, PTEN, PTPN12, PRKAG2, PRKCZ, PRKD3, SLCO1B3), type 2 diabetes (T2D) (AKT3, PRKAG2, PRKCZ, PRKD3), and CV signaling (AKT3, HDAC4, PRKAG2, PRKCZ, PRKD3).

\section{DNA methylation and biochemical parameters}

We further investigated the possible relationship between $\Delta$ methylation of these 19 genes related to $\mathrm{CV}$ health and changes in the four biochemical parameters modified by the $n-3$ FA supplementation $(\Delta T G, \Delta T C$, $\triangle \mathrm{TC} / \mathrm{HDL}-\mathrm{C}$, and $\Delta$ Glucose). As shown in Table 4, $\triangle$ methylation of cg00011856 (IGFBP5) was positively correlated with $\triangle \mathrm{TG}(r=0.39, p=0.023)$ while $\Delta$ methylation of $\operatorname{cg} 05655647$ (ATF1) was negatively correlated with $\triangle \mathrm{TG}(r=-0.35, p=0.047)$. The $\Delta$ methylation of cg00011856 (IGFBP5) and cg24455383 (AKT3) were positively correlated with $\triangle \mathrm{TC} / \mathrm{HDL}-\mathrm{C}(r=0.36, p=$ 0.042 and $r=0.42, p=0.016$, respectively). The $\Delta$ methylation of cg15656521 (HDAC4) was positively correlated with $\Delta$ Glucose $(r=0.35, p=0.043)$. There was no significant correlation with $\Delta \mathrm{TC}$. After adjustments for age, sex, and body mass index (BMI), the correlation between $\Delta$ methylation of cg00011856 and $\Delta \mathrm{TG}$ remained significant $(r=0.42, p=0.020)$ as well as the one between $\Delta$ methylation of cg24455383 and $\Delta$ TC/HDL-C $(r=0.40$, $p=0.031$ ).

Using previous data on gene expression in the same cohort [27], we performed further analyses to test whether correlations between $\Delta$ methylation and changes in biochemical parameters may potentially be attributable to changes in expression levels ( $\Delta$ expression). We first correlated $\triangle$ methylation and $\triangle$ expression for these four genes (ATF1, AKT3, HDAC4, and IGFBP5). ATF1 and HDAC4 showed significant correlation between $\Delta$ methylation and $\Delta$ expression after adjustments for the

Table 3 CV health-related overrepresented pathways identified from differential methylation analysis following an n-3 FA supplementation

\begin{tabular}{|c|c|c|}
\hline IPA canonical pathways & $P$ value & Differentially methylated genes \\
\hline Tumoricidal function of hepatic natural killer cells ${ }^{a}$ & 0.0017 & BAX, CASP6, FAS \\
\hline RAR activation ${ }^{a}$ & 0.0035 & AKT3, DHRS9, PRKAG2, PRKCZ, PRKD3, PTEN, TRIM24 \\
\hline VDR/RXR activation ${ }^{\mathrm{b}}$ & 0.0078 & IGFBP5, KLKG, PRKCZ, PRKD3 \\
\hline Fcy receptor-mediated phagocytosis in macrophages and monocytes ${ }^{a}$ & 0.017 & AKT3, PRKCZ, PRKD3, PTEN \\
\hline D-myo-inositol-5-phosphate metabolism ${ }^{\text {b }}$ & 0.018 & NUDT3, PLCH1, PPP2R5E, PTEN, PTPN12 \\
\hline Nitric oxide signaling in the cardiovascular system ${ }^{c}$ & 0.026 & AKT3, PRKAG2, PRKCZ, PRKD3 \\
\hline IL-3 signaling ${ }^{\mathrm{a}}$ & 0.033 & AKT3, PRKCZ, PRKD3 \\
\hline PXR/RXR activation ${ }^{\mathrm{b}}$ & 0.035 & AKT3, SLCO1B3, PRKAG2 \\
\hline LPS-stimulated MAPK signaling ${ }^{a}$ & 0.035 & ATF1, PRKCZ, PRKD3 \\
\hline NF-kB activation by viruses ${ }^{a}$ & 0.037 & AKT3, PRKCZ, PRKD3 \\
\hline CCR5 signaling in nacrophages ${ }^{a}$ & 0.037 & FAS, PRKCZ, PRKD3 \\
\hline Role of NFAT in cardiac hypertrophy ${ }^{c}$ & 0.038 & AKT3, HDAC4, PRKAG2, PRKCZ, PRKD3 \\
\hline P2Y purigenic receptor signaling pathway ${ }^{c}$ & 0.040 & AKT3, PRKAG2, PRKCZ, PRKD3 \\
\hline Cytotoxic T lymphocyte-mediated apoptosis of target cells ${ }^{a}$ & 0.040 & FAS, CASPG \\
\hline PI3K signaling in B lymphocytes ${ }^{a}$ & 0.044 & AKT3, ATF1, PRKCZ, PTEN \\
\hline Type II diabetes mellitus signaling ${ }^{d}$ & 0.049 & AKT3, PRKAG2, PRKCZ, PRKD3 \\
\hline
\end{tabular}

Pathways related to the following: ${ }^{a}$ Inflammatory and immune response $(n=9) ;{ }^{\mathrm{b}}$ Lipid metabolism $(n=3) ;{ }^{\mathrm{C}}$ Cardiovascular signaling $(n=3) ;{ }^{\mathrm{d}}$ Diabetes $(n=1)$ 
Table 4 Significant correlations between changes in biochemical parameters and changes in methylation levels following an n-3 FA supplementation

\begin{tabular}{llll}
\hline CpG site ID (gene, position $\left.{ }^{\mathrm{a}}\right)$ & $\Delta$ Triglyceride & $\Delta$ TC/HDL-C & $\Delta$ Glucose \\
\hline$\triangle \mathrm{cg} 00011856($ IGFBP5, Chr2:217560946) & $0.39(0.023)^{\mathrm{b}}$ & $0.36(0.042)$ & - \\
$\triangle \mathrm{cg} 05655647$ (ATF1, Chr12:51157023) & $-0.35(0.047)$ & - & - \\
$\Delta \mathrm{cg} 24455383($ AKT3, Chr1:243736307) & - & $0.42(0.016)^{\mathrm{b}}$ & - \\
$\triangle \mathrm{cg} 15656521$ (HDAC4, Chr2:239970617) & - & - & $0.35(0.043)$ \\
\hline
\end{tabular}

Results are $R$ ( $P$ value)

Abbreviations: HDL-C high-density lipoprotein cholesterol, TC total cholesterol

${ }^{a}$ All positions are from the Genome Build 37

${ }^{\mathrm{b}}$ Correlation remains significant after adjustments for age, sex, and body mass index

effects of age, sex, and BMI $(r=-0.44, p=0.02$ and $r=$ $0.47, p=0.01)$. We then correlated $\triangle$ expression of ATF1 and $H D A C 4$ with $\triangle \mathrm{TG}$ and $\triangle$ Glucose, respectively. However, we found no significant association.

\section{Discussion}

The aim of this study was to investigate DNA methylation changes following n-3 FA supplementation and identify potentially altered biological pathways. We first observed that a 6-week supplementation with $3 \mathrm{~g}$ of $\mathrm{n}-3$ FAs per day was associated with a decrease in n-6 FAs and an increase in n-3 FAs in plasma phospholipids of 36 overweight and obese adults. The n-3 FA supplementation also decreased plasma TG, TC, and the ratio TC/ HDL-C, while increasing plasma glucose concentrations. The slight increase in glucose concentrations was similar to the one reported in the entire cohort [25]. Studies have reported conflicting results on the effect of n-3 FAs on blood glucose concentrations [28]. There is a large variability in plasma glucose response to n-3 FAs with effects varying from $1.61 \mathrm{mmol} / \mathrm{L}$ net reduction to $1.4 \mathrm{mmol} / \mathrm{L}$ net increase [29].

Using a genome-wide methylation analysis, we identified differences in CpG sites methylation levels following an n-3 FA supplementation. As previously mentioned, only few studies were conducted on the impact of n-3 FAs on DNA methylation in humans [19-24, 30]. Our results are in agreement with these studies suggesting that EPA and DHA can modulate DNA methylation levels. Moreover, we identified overrepresented pathways from differentially methylated genes. More precisely, pathway analysis revealed 16 overrepresented pathways related to $\mathrm{CV}$ health. These results are in line with previously reported effects of n-3 FAs and DNA methylation on $\mathrm{CV}$ health. Indeed, n-3 FAs have beneficial effects on CV risk factors [1-9], even if they are not associated with CV disease events [31]. Moreover, epidemiological studies have reported the association between global DNA methylation levels and prevalence of CV diseases $[17,18]$.

Among the 16 pathways related to $\mathrm{CV}$ health, nine were related to inflammatory and immune response which may be in line with potential anti-inflammatory effects of n-3 FAs, more particularly DHA [5, 32]. Studies have also reported the link between DNA methylation and inflammation. A recent study in the GOLDN study and the ENCODE consortium reported that higher erythrocyte total n-3 FAs was associated with lower cg01770232 methylation (IL-6) and lower plasma IL-6 concentration [30]. The hypomethylation of longinterspersed element-1 was also associated with higher serum vascular cell adhesion molecule- 1 in elderly men [33]. Moreover, three pathways were related to lipid metabolism, which is in accordance with known hypotriglyceridemic effects of n-3 FAs [1]. Methylation levels of one CpG site in $A P O E$ and one in $A B C A 1$ were negatively associated with plasma TG and HDL-C levels, respectively, in the GOLDN study [34, 35]. DNA methylation is also implicated in the regulation of atherosclerosis. Indeed, genome-wide DNA methylation changes (mainly hypermethylation) occur during the onset and progression of atherosclerotic lesions in humans $[36,37]$. A total of three pathways were related to cardiovascular signaling. Indeed, reported antiplatelet effects of n-3 FAs [38] are consistent with the overrepresentation of the nitric oxide signaling in the cardiovascular system pathway identified herein. Finally, one pathway was related to T2D, which may be in line with potential, but yet controversial, effects of n-3 FAs on glucose homeostasis [39]. Data mining analysis suggests a role of epigenetic factors in the pathogenesis of T2D [40]. Differential methylation profiles in pancreatic islets from T2D and non-diabetic subjects were also identified thus suggesting a role of DNA methylation in pathogenesis of T2D [41]. Moreover, blood DNA methylation of some $\mathrm{CpG}$ sites have been associated with blood glucose concentrations in an epigenome-wide association study [42]. All together, these finding suggest a possible link between n-3 FAs, DNA methylation, and $\mathrm{CV}$ risk factors.

Among differentially methylated genes, some were also of particular interest in the field of $\mathrm{CV}$ health. For example, a genetic variation in the $F A S$ gene was associated with an increased occurrence of myocardial 
infarction in Japanese subjects [43]. Interestingly, FAS methylation levels were significantly associated with n-3 FA intakes in Yup'ik Alaska Native individuals [23]. Moreover, a total of four CpG sites showed correlations between changes in their methylation levels and changes in biochemical parameters following the supplementation. First, $\triangle$ methylation of cg15656521 in HDAC4 gene was positively correlated with $\triangle$ glucose. HDAC4 gene encodes for a histone deacetylase 4 that is a signaldependent modulator of transcription with role in muscle differentiation and neuronal survival [44]. HDAC4 methylation was inversely associated with $\mathrm{n}-3$ FAs in whole blood of men [45]. HDAC4 also downregulates GLUT4 transcription in cultured adipocytes and fasting mice [46]. Interestingly, Benton et al. reported a robust inverse correlation between changes in fasting glucose and changes in methylation of HDAC4 (cg26078407) in subcutaneous fat [47]. However, they did not test for association with cg15656521 since it was not differentially methylated in their cohort after gastric bypass surgery [47]. Second, $\triangle$ methylation of cg00011856 in IGFBP5 gene was positively correlated with $\triangle \mathrm{TG}$ (even after adjustments for age, sex, and BMI) and with $\triangle \mathrm{TC} /$ HDL-C. IGFBP5 gene encodes for insulin-like growth factor binding protein 5 [48]. Young patients with coronary heart disease have significantly higher serum IGFBP5 than age- and BMI-matched controls [49]. A study in arthritic rats demonstrated that EPA increases IGFBP5 mRNA in the gastrocnemius muscle [50]. Another study found higher IGFBP5 expression in the liver of beef fed with $\mathrm{n}-3$ FAs compared to control diet [51]. Unfortunately, we cannot verify if the n-3 FA supplementation increases IGFBP5 expression in our sample since it is not expressed in blood [52], but it would be interesting to look at its expression in the liver after an n-3 FA supplementation. Third, $\Delta$ methylation of cg24455383 in AKT3 was positively correlated with $\triangle \mathrm{TC}$-HDL-C, even after adjustments. AKT3 gene encodes for an AKT serine/threonine kinase 3 stimulated by insulin and growth factors $[53,54]$. It is likely involved in insulin-stimulated glucose transport in the human skeletal muscles [55]. AKT3 also has anti-atherogenic properties due to its capacity to inhibit macrophages foam cells formation by reducing lipoprotein uptake and promoting ACAT-1 degradation $[56,57]$. AKT signaling has also been shown to regulate lipid metabolism through phosphorylation and inhibition of GSK3 [58]. The association between changes in $A K T 3$ methylation and changes in TC/HDL-C ratio reported herein may be plausible since $A K T 3$ seems to be involved in lipid metabolism. Finally, $\triangle$ methylation of cg05655647 in ATF1 gene was negatively correlated with $\triangle \mathrm{TG}$. The ATF1 gene encodes for the activating transcription factor 1 that leads to the production of atheroprotective macrophages [59, 60]. SNPs within this gene were also associated with an increased risk of essential hypertension in a case-control study [61]. Data are insufficient to propose a mechanism of action between methylation of ATF1 and plasma TG levels. Nevertheless, we could hypothesize that methylation of these genes may play a role in the effects of $n-3$ FAs on $C V$ health since they are modulated by n-3 FAs, they have been associated with CV health in pathway analysis, and they are correlated with changes in biochemical parameters. However, we acknowledge that our study design does not allow us to prove or investigate causality between n-3 FA supplementation, DNA methylation, and CV disease risk factors. Moreover, we were not able to investigate the potential link between $\triangle$ methylation of ATF1, AKT3, IGFBP5, and $H D A C 4$ and changes in biochemical parameters using $\Delta$ expression. At this time, we cannot rule out the possibility that the small sample size or low $\Delta$ expression values of these genes limit our statistical power to detect significant associations. This possible link does not seem to be due either to surrounding SNPs since they affected $\Delta$ methylation of only two $\mathrm{CpG}$ sites among the 308 CpG sites.

The present study has some limitations. DNA methylation levels are specific to the type of cell and tissue [62]. These methylation profiles in blood leukocytes might not represent DNA methylation in other tissues even though these patterns are globally conserved across tissues [63-65]. Pathway analysis has certain methodological considerations. As part of Genetic Analysis Workshop 18, seven research groups raised the fact that annotation of genetic variants is inconsistent across databases, incomplete and biased toward known genes [66]. Moreover, insufficient statistical power is an issue in pathway analyses [66]. Thus, these results need to be validated in larger and independent studies considering that replication remains the gold standard to establish validity of the findings. Finally, the sample size is relatively small so these results need to be validated in larger, independent studies.

\section{Conclusions}

In conclusion, the present data provide key differences in blood leukocytes DNA methylation levels of subjects following n-3 FA supplementation, which provides new, potential insights on novel genes and metabolic pathways underlying the effects of n-3 FAs on the CV risk profile. Further studies in larger, independent samples are required to unveil potential functional mechanisms underlying metabolic improvements with n-3 FA supplementation.

\section{Methods}

\section{Study population and study design}

The present study is based on a subsample of a larger intervention study that aimed at studying the inter- 
individual variability in TG response to an n-3 FA supplementation as previously described [2]. A total of 254 subjects from the greater Quebec City metropolitan area (Canada) were recruited between September 2009 and December 2011. In total, 210 subjects completed the intervention. Non-smoker subjects, aged between 18 and 50 , with a BMI between 25 and $40 \mathrm{~kg} / \mathrm{m}^{2}$, and not taking any lipid-lowering medication were included. Subjects were excluded if they had taken n-3 FA supplementation at least 6 months prior to the intervention or had been diagnosed with any metabolic disorder. A subset of 36 subjects who first completed the study (equal proportion of men and women) was selected for the purpose of this genome-wide DNA methylation analysis.

First, subjects received dietary instructions by a trained registered dietician to achieve recommendations from Canada's Food Guide during a 2-week run-in period [67]. After this period, subjects receive a bottle containing fish oil capsules needed for the following 6 weeks. They were invited to take five capsules per day providing a total of $3 \mathrm{~g}$ of $\mathrm{n}-3$ FAs (including 1.9-2.2 $\mathrm{g}$ of EPA and $1.1 \mathrm{~g}$ of DHA) per day. Compliance was assessed by the count of remaining capsules and by measuring EPA and DHA in plasma phospholipids. Details on the study design have been described elsewhere [2]. The experimental protocol was approved by the Ethics Committees of Laval University Hospital Research Center and Laval University. This trial was registered at clinicaltrials.gov as NCT01343342.

\section{Anthropometric and metabolic measurements}

Body weight, waist girth, and height were measured according to the procedures recommended by the Airlie Conference [68] and were taken before the run-in period as well as before and after the supplementation period. Blood samples were collected from an antecubital vein into vacutainer tubes containing EDTA after 12-h overnight fast and 48-h alcohol abstinence before the run-in period to identify and exclude participants with metabolic disorders. Afterwards, the selected participants had blood samples taken before and after the supplementation period. Plasma was separated by centrifugation (2500 g for $10 \mathrm{~min}$ at $4{ }^{\circ} \mathrm{C}$ ), and samples were aliquoted and frozen $\left(-80{ }^{\circ} \mathrm{C}\right)$ for subsequent analyses. Enzymatic assays were used to measure plasma TC and TG concentrations $[69,70]$. Precipitation of very-low density lipoprotein (VLDL) and low-density lipoprotein (LDL) particles in the infranatant with heparin manganese chloride generated the HDL-C fraction [71]. LDL cholesterol (LDL-C) was calculated with the Friedewald formula [72]. Using a sensitive assay, plasma C-reactive protein (CRP) was measured by nephelometry (Prospec equipment Behring) [73].

\section{DNA extraction and DNA methylation analysis}

We extracted genomic DNA from blood leukocytes using the GenElute Blood Genomic DNA kit (Sigma-Aldrich, St. Louis, MO, USA) for the 72 samples: 36 samples before and 36 samples after the supplementation. DNA was quantified using both NanoDrop Spectrophotometer (Thermo Scientific, Wilmington, DE, USA) and PicoGreen DNA methods. McGill University and Genome Quebec Innovation Center (Montreal, QC, Canada) conducted the bisulfite conversion and quantitative DNA methylation analysis using Infinium HumanMethylation450 array (Illumina, San Diego, CA, USA). Three samples (one sample before and two samples after supplementation) were excluded from microarray analysis following quality control steps (bisulfite conversion, extension, staining, hybridization, target removal, negative and nonpolymorphic control probes).

We used Illumina GenomeStudio software version 2011.1 and the Methylation Module to analyze methylation data on 485,577 CpG sites. Methylation levels (varying from 0 to 1 ) were estimated as the proportion of total signal intensity from methylated-specific probe. To avoid false positives, probes with a detection $P$ value $>0.05$ in more than $10 \%$ of samples were removed. Probes on the $\mathrm{X}$ and $\mathrm{Y}$ chromosomes were also removed to eliminate gender bias. Thus, 472,715 probes were considered in differential methylation analysis. Differences in methylation levels before and after n-3 FA supplementation were tested using the Illumina Custom model in GenomeStudio software. FDR-corrected DiffScores were computed to account for multiple testing and limit false positives. We established significant differences following n-3 FA supplementation with a FDRcorrected DiffScore $\geq|13| \sim P \leq 0.05$.

\section{Pathway analyses}

We used the IPA system (Ingenuity Systems, www.ingenuity.com) to analyze potentially modified pathways from differentially methylated genes following the supplementation. Using a right-tailed Fisher's exact test, IPA measured the likelihood that pathways were overrepresented among the list of significant differentially methylated genes.

\section{Statistical analyses}

We tested potential relationship between presupplementation methylation levels and $\Delta$ methylation of the $308 \mathrm{CpG}$ sites and surrounding SNPs $( \pm 1 \mathrm{~kb})$. We used results from a recent GWAS done by our group in the same cohort $(n=141)$ [26]. We considered only SNPs located at $\pm 1 \mathrm{~kb}$ from CpG sites and with a minor allele frequency $\geq 1 \%$. We tested 652 associations using analysis of variance (general linear model, type III sum of squares) and adjusted for the 
effects of age, sex, and BMI. We used a Bonferroni correction for multiple testing; thus, associations with a $P$ value $\leq 7.67 \times 10^{-5}(0.05 / 652)$ were considered significant.

Biochemical parameters are expressed as means \pm SD. We used a paired Student's $t$ test to test differences in biochemical parameters before and after the supplementation. Variables not normally distributed were $\log _{10}$ transformed before analyses. $\Delta$ methylation of $\mathrm{CpG}$ sites $(n=20)$ within genes in the 16 pathways related to CV health were defined as post-supplementation methylation levels minus pre-supplementation methylation levels. Changes in the four significantly modulated biochemical parameters $(\Delta \mathrm{TG}, \Delta \mathrm{TC}, \Delta \mathrm{TC} / \mathrm{HDL}-\mathrm{C}$, and $\Delta$ Glucose) were defined as (post-supplementation values minus presupplementation values)/ pre-supplementation values to account for baseline values. Potential relationships between $\Delta$ methylation, $\Delta$ expression, and changes in biochemical parameters $(\Delta \mathrm{TG}, \Delta \mathrm{TC}, \Delta \mathrm{TC} / \mathrm{HDL}-\mathrm{C}$, and $\Delta$ Glucose) were investigated using Pearson's correlation. We also accounted for potential confounding effects of age, sex, and BMI in correlations. Statistical analyses were conducted using SAS software version 9.3 (SAS Institute, Cary, NC, USA).

\section{Additional files}

Additional file 1: Differentially methylated probes after the n-3 FA supplementation. Description of data: List of all 308 differentially methylated probes (FDR-corrected DiffScore $\geq|13| \sim P \leq 0.05$ ). (XLSX $42 \mathrm{~kb})$

Additional file 2: Overrepresented pathways identified from differential methylation analysis following an n-3 FA supplementation. Description of data: Table describing all 55 significant overrepresented pathways identified from methylation analysis (IPA canonical pathways, associated $P$ value, and list of differentially methylated genes). (DOCX $21 \mathrm{~kb}$ )

\section{Abbreviations}

BMI: Body mass index; CpG: Cytosine-phosphate-guanine; CV: Cardiovascular; DHA: Docosahexaenoic acid; EPA: Eicosapentaenoic acid; FDR: False discovery rate; HDL-C: High-density lipoprotein cholesterol; IPA: Ingenuity Pathway Analysis; n-3 FAs: Omega-3 fatty acids; SNPs: Single-nucleotide polymorphisms; T2D: Type 2 diabetes; TC: Total cholesterol; TG: Triglyceride

\section{Acknowledgements}

We would like to thank Véronique Garneau, Ann-Marie Paradis, Élisabeth Thifault, Karelle Dugas-Bourdage, Catherine Ouellette, and Annie Bouchard-Mercier, who contributed to the success of this study. We also thank Catherine Raymond for the laboratory work.

\section{Funding}

This work was supported by a grant from Canadian Institutes of Health Research (CIHR)-(MOP-110975). IR holds a Junior 1 Research Scholar from the Fonds de Recherche du Québec-Santé (FRQS). BLT is a recipient of a scholarship from FRQS. MCV is Tier 1 Canada Research Chair in Genomics Applied to Nutrition and Health.

\section{Availability of data and materials}

Methylation datasets supporting the conclusion of this article are available in the Gene Expression Omnibus (GEO) database, www.ncbi.nlm.nih.gov/geo (GSE98056).

\section{Authors' contributions}

Each author contribution to work: IR, SL, and MCV designed the research; PC was responsible for the medical follow-up; BLT and FG conducted the research and performed the statistical analyses; BLT wrote the paper; and BLT and MCV have primary responsibility for the final content. All authors read and approved the final manuscript.

\section{Competing interests}

The authors declare that they have no competing interests.

\section{Consent for publication}

Not applicable.

\section{Ethics approval and consent to participate}

All participants signed an informed consent document. This trial was registered at clinicaltrials.gov as NCT01343342.

\section{Publisher's Note}

Springer Nature remains neutral with regard to jurisdictional claims in published maps and institutional affiliations.

Received: 15 December 2016 Accepted: 14 April 2017

Published online: 26 April 2017

\section{References}

1. Caslake MJ, Miles EA, Kofler BM, Lietz G, Curtis P, Armah CK, Kimber AC, Grew JP, Farrell L, Stannard J, et al. Effect of sex and genotype on cardiovascular biomarker response to fish oils: the FINGEN Study. Am J Clin Nutr. 2008:88:618-29.

2. Cormier H, Rudkowska I, Paradis AM, Thifault E, Garneau V, Lemieux S, Couture $P$, Vohl MC. Association between polymorphisms in the fatty acid desaturase gene cluster and the plasma triacylglycerol response to an n-3 PUFA supplementation. Nutrients. 2012:4:1026-41.

3. Farzaneh-Far R, Harris WS, Garg S, Na B, Whooley MA. Inverse association of erythrocyte $\mathrm{n}-3$ fatty acid levels with inflammatory biomarkers in patients with stable coronary artery disease: The Heart and Soul Study. Atherosclerosis. 2009;205:538-43.

4. Madsen T, Christensen JH, Blom M, Schmidt EB. The effect of dietary n-3 fatty acids on serum concentrations of $\mathrm{C}$-reactive protein: a dose-response study. Br J Nutr. 2003;89:517-22.

5. Allaire J, Couture P, Leclerc M, Charest A, Marin J, Lepine MC, Talbot D, Tchernof A, Lamarche B. A randomized, crossover, head-to-head comparison of EPA and DHA supplementation to reduce inflammation markers in men and women: Comparing EPA to DHA (ComparED) Study. Am J Clin Nutr. 2016;104(2):280-7.

6. Harris WS, Miller M, Tighe AP, Davidson MH, Schaefer EJ. Omega-3 fatty acids and coronary heart disease risk: clinical and mechanistic perspectives. Atherosclerosis. 2008;197:12-24.

7. Xin W, Wei W, Li XY. Short-term effects of fish-oil supplementation on heart rate variability in humans: a meta-analysis of randomized controlled trials. Am J Clin Nutr. 2013;97:926-35.

8. von Schacky C, Fischer S, Weber PC. Long-term effects of dietary marine omega-3 fatty acids upon plasma and cellular lipids, platelet function, and eicosanoid formation in humans. J Clin Invest. 1985;76:1626-31.

9. Thies F, Garry JM, Yaqoob P, Rerkasem K, Williams J, Shearman CP, Gallagher PJ, Calder PC, Grimble RF. Association of n-3 polyunsaturated fatty acids with stability of atherosclerotic plaques: a randomised controlled trial. Lancet. 2003;361:477-85.

10. Burdge GC, Lillycrop KA. Fatty acids and epigenetics. Curr Opin Clin Nutr Metab Care. 2014;17:156-61.

11. Kulkarni A, Dangat K, Kale A, Sable P, Chavan-Gautam P, Joshi S. Effects of altered maternal folic acid, vitamin B12 and docosahexaenoic acid on placental global DNA methylation patterns in Wistar rats. PLoS One. 2011;6:e17706.

12. Daxinger $L$, Whitelaw $E$. Understanding transgenerational epigenetic inheritance via the gametes in mammals. Nat Rev Genet. 2012;13:153-62.

13. Friso S, Pizzolo F, Choi SW, Guarini P, Castagna A, Ravagnani V, Carletto A, Pattini P, Corrocher R, Olivieri O. Epigenetic control of 11 betahydroxysteroid dehydrogenase 2 gene promoter is related to human hypertension. Atherosclerosis. 2008;199:323-7.

14. Lund G, Andersson L, Lauria M, Lindholm M, Fraga MF, Villar-Garea A Ballestar E, Esteller M, Zaina S. DNA methylation polymorphisms precede 
any histological sign of atherosclerosis in mice lacking apolipoprotein E. J Biol Chem. 2004;279:29147-54.

15. Turunen MP, Aavik E, Yla-Herttuala S. Epigenetics and atherosclerosis. Biochim Biophys Acta. 1790;2009:886-91.

16. Makar KW, Wilson CB. DNA methylation is a nonredundant repressor of the Th2 effector program. J Immunol. 2004;173:4402-6.

17. Baccarelli A, Wright R, Bollati V, Litonjua A, Zanobetti A, Tarantini L, Sparrow D, Vokonas P, Schwartz J. Ischemic heart disease and stroke in relation to blood DNA methylation. Epidemiology. 2010;21:819-28.

18. Kim M, Long Tl, Arakawa K, Wang R, Yu MC, Laird PW. DNA methylation as a biomarker for cardiovascular disease risk. PLoS One. 2010;5:e9692.

19. Lind MV, Martino D, Harslof LB, Kyjovska ZO, Kristensen M, Lauritzen L. Genome-wide identification of mononuclear cell DNA methylation sites potentially affected by fish oil supplementation in young infants: a pilot study. Prostaglandins Leukot Essent Fatty Acids. 2015;101:1-7.

20. Voisin S, Almen MS, Moschonis G, Chrousos GP, Manios Y, Schioth HB. Dietary fat quality impacts genome-wide DNA methylation patterns in a crosssectional study of Greek preadolescents. Eur J Hum Genet. 2015;23:654-62.

21. Hoile SP, Clarke-Harris R, Huang RC, Calder PC, Mori TA, Beilin LJ, Lillycrop KA, Burdge GC. Supplementation with N-3 long-chain polyunsaturated fatty acids or olive oil in men and women with renal disease induces differential changes in the DNA methylation of FADS2 and ELOVL5 in peripheral blood mononuclear cells. PLoS One. 2014;9:e109896.

22. DoAmaral $\mathrm{CL}$, Milagro Fl, Curi R, Martinez JA. DNA methylation pattern in overweight women under an energy-restricted diet supplemented with fish oil. Biomed Res Int. 2014;2014:675021.

23. Aslibekyan S, Wiener HW, Havel PJ, Stanhope KL, O'Brien DM, Hopkins SE, Absher DM, Tiwari HK, Boyer BB. DNA methylation patterns are associated with n-3 fatty acid intake in Yup'ik people. J Nutr. 2014;144:425-30.

24. Lee HS, Barraza-Villarreal A, Hernandez-Vargas H, Sly PD, Biessy C, Ramakrishnan U, Romieu I, Herceg Z. Modulation of DNA methylation states and infant immune system by dietary supplementation with omega-3 PUFA during pregnancy in an intervention study. Am J Clin Nutr. 2013;98:480-7.

25. Thifault E, Cormier H, Bouchard-Mercier A, Rudkowska I, Paradis AM, Garneau V, Ouellette C, Lemieux S, Couture P, Vohl MC. Effects of age, sex, body mass index and APOE genotype on cardiovascular biomarker response to an n-3 polyunsaturated fatty acid supplementation. J Nutrigenet Nutrigenomics. 2013;6:73-82.

26. Rudkowska I, Guenard F, Julien P, Couture P, Lemieux S, Barbier O, Calder PC, Minihane AM, Vohl MC. Genome-wide association study of the plasma triglyceride response to an $n-3$ polyunsaturated fatty acid supplementation. J Lipid Res. 2014;55:1245-53.

27. Rudkowska I, Paradis AM, Thifault E, Julien P, Barbier O, Couture P, Lemieux $S$, Vohl MC. Differences in metabolomic and transcriptomic profiles between responders and non-responders to an $n-3$ polyunsaturated fatty acids (PUFAs) supplementation. Genes Nutr. 2013;8:411-23.

28. Clark LF, Thivierge MC, Kidd CA, McGeoch SC, Abraham P, Pearson DW, Horgan GW, Holtrop G, Thies F, Lobley GE. Fish oil supplemented for 9 months does not improve glycaemic control or insulin sensitivity in subjects with impaired glucose regulation: a parallel randomised controlled trial. Br J Nutr. 2016;115:75-86.

29. Balk EM, Lichtenstein AH, Chung M, Kupelnick B, Chew P, Lau J. Effects of omega-3 fatty acids on serum markers of cardiovascular disease risk: a systematic review. Atherosclerosis. 2006;189:19-30.

30. Ma Y, Smith CE, Lai CQ, Irvin MR, Parnell LD, Lee YC, Pham LD, Aslibekyan S, Claas SA, Tsai MY, et al. The effects of omega-3 polyunsaturated fatty acids and genetic variants on methylation levels of the interleukin- 6 gene promoter. Mol Nutr Food Res. 2016;60:410-9.

31. Rizos EC, Ntzani EE, Bika E, Kostapanos MS, Elisaf MS. Association between omega-3 fatty acid supplementation and risk of major cardiovascular disease events: a systematic review and meta-analysis. JAMA. 2012;308:1024-33.

32. Li K, Huang T, Zheng J, Wu K, Li D. Effect of marine-derived n-3 polyunsaturated fatty acids on C-reactive protein, interleukin 6 and tumor necrosis factor alpha: a meta-analysis. PLoS One. 2014;9:e88103.

33. Baccarelli A, Tarantini L, Wright RO, Bollati V, Litonjua AA, Zanobetti A, Sparrow D, Vokonas PS, Schwartz J. Repetitive element DNA methylation and circulating endothelial and inflammation markers in the VA normative aging study. Epigenetics. 2010;5:222-8.

34. Naj AC, Jun G, Reitz C, Kunkle BW, Perry W, Park YS, Beecham GW, Rajbhandary RA, Hamilton-Nelson KL, Wang LS, et al. Effects of multiple genetic loci on age at onset in late-onset Alzheimer disease: a genomewide association study. JAMA Neurol. 2014;71:1394-404.

35. Ma Y, Follis JL, Smith CE, Tanaka T, Manichaikul AW, Chu AY, Samieri C, Zhou X, Guan W, Wang L, et al. Interaction of methylation-related genetic variants with circulating fatty acids on plasma lipids: a meta-analysis of 7 studies and methylation analysis of 3 studies in the Cohorts for Heart and Aging Research in Genomic Epidemiology consortium. Am J Clin Nutr. 2016;103:567-78.

36. Zaina S, Heyn H, Carmona FJ, Varol N, Sayols S, Condom E, Ramirez-Ruz J, Gomez A, Goncalves I, Moran S, Esteller M. DNA methylation map of human atherosclerosis. Circ Cardiovasc Genet. 2014;7:692-700.

37. Valencia-Morales Mdel P, Zaina S, Heyn H, Carmona FJ, Varol N, Sayols S, Condom E, Ramirez-Ruz J, Gomez A, Moran S, et al. The DNA methylation drift of the atherosclerotic aorta increases with lesion progression. BMC Med Genomics. 2015;8:7.

38. Larson MK, Shearer GC, Ashmore JH, Anderson-Daniels JM, Graslie EL, Tholen JT, Vogelaar JL, Korth AJ, Nareddy V, Sprehe M, Harris WS. Omega-3 fatty acids modulate collagen signaling in human platelets. Prostaglandins Leukot Essent Fatty Acids. 2011;84:93-8.

39. Akinkuolie AO, Ngwa JS, Meigs JB, Djousse L. Omega-3 polyunsaturated fatty acid and insulin sensitivity: a meta-analysis of randomized controlled trials. Clin Nutr. 2011;30:702-7.

40. Wren JD, Garner HR. Data-mining analysis suggests an epigenetic pathogenesis for type 2 diabetes. J Biomed Biotechnol. 2005;2005:104-12.

41. Volkmar M, Dedeurwaerder S, Cunha DA, Ndlovu MN, Defrance M, Deplus R, Calonne E, Volkmar U, Igoillo-Esteve M, Naamane N, et al. DNA methylation profiling identifies epigenetic dysregulation in pancreatic islets from type 2 diabetic patients. EMBO J. 2012;31:1405-26.

42. Kriebel J, Herder C, Rathmann W, Wahl S, Kunze S, Molnos S, Volkova N, Schramm K, Carstensen-Kirberg M, Waldenberger M, et al. Association between DNA methylation in whole blood and measures of glucose metabolism: KORA F4 Study. PLoS One. 2016;11:e0152314.

43. Hanasaki H, Takemura Y, Fukuo K, Ohishi M, Onishi M, Yasuda O, Katsuya T, Awata N, Kato N, Ogihara T, Rakugi H. Fas promoter region gene polymorphism is associated with an increased risk for myocardial infarction. Hypertens Res. 2009;32:261-4.

44. Mihaylova MM, Vasquez DS, Ravnskjaer K, Denechaud PD, Yu RT, Alvarez JG, Downes M, Evans RM, Montminy M, Shaw RJ. Class lla histone deacetylases are hormone-activated regulators of FOXO and mammalian glucose homeostasis. Cell. 2011:145:607-21.

45. de la Rocha C, Perez-Mojica JE, Leon SZ, Cervantes-Paz B, Tristan-Flores FE, Rodriguez-Rios D, Molina-Torres J, Ramirez-Chavez E, Alvarado-Caudillo Y, Carmona FJ, et al. Associations between whole peripheral blood fatty acids and DNA methylation in humans. Sci Rep. 2016;6:25867.

46. Weems JC, Griesel BA, Olson AL. Class II histone deacetylases downregulate GLUT4 transcription in response to increased CAMP signaling in cultured adipocytes and fasting mice. Diabetes. 2012;61:1404-14.

47. Benton MC, Johnstone A, Eccles D, Harmon B, Hayes MT, Lea RA, Griffiths L, Hoffman EP, Stubbs RS, Macartney-Coxson D. An analysis of DNA methylation in human adipose tissue reveals differential modification of obesity genes before and after gastric bypass and weight loss. Genome Biol. 2015;16:8.

48. Beattie J, Allan GJ, Lochrie JD, Flint DJ. Insulin-like growth factor-binding protein-5 (IGFBP-5): a critical member of the IGF axis. Biochem J. 2006;395:1-19.

49. Fischer F, Schulte H, Mohan S, Tataru MC, Kohler E, Assmann G, von Eckardstein A. Associations of insulin-like growth factors, insulin-like growth factor binding proteins and acid-labile subunit with coronary heart disease. Clin Endocrinol (Oxf). 2004;61:595-602.

50. Castillero E, Lopez-Menduina M, Martin Al, Villanua MA, Lopez-Calderon A. Comparison of the effects of the n-3 polyunsaturated fatty acid eicosapentaenoic and fenofibrate on the inhibitory effect of arthritis on IGF1. J Endocrinol. 2011;210:361-8.

51. Coyne GS, Kenny DA, Waters SM. Effect of dietary n-3 polyunsaturated fatty acid supplementation on bovine uterine endometrial and hepatic gene expression of the insulin-like growth factor system. Theriogenology. 2011;75:500-12.

52. Liu X, Yu X, Zack DJ, Zhu H, Qian J. TiGER: a database for tissue-specific gene expression and regulation. BMC Bioinformatics. 2008;9:271.

53. Gai D, Haan E, Scholar M, Nicholl J, Yu S. Phenotypes of AKT3 deletion: a case report and literature review. Am J Med Genet A. 2015;167A:174-9.

54. Sandirasegarane L, Kester M. Enhanced stimulation of Akt-3/protein kinase B-gamma in human aortic smooth muscle cells. Biochem Biophys Res Commun. 2001;283:158-63. 
55. Brozinick Jr JT, Roberts BR, Dohm GL. Defective signaling through Akt-2 and -3 but not Akt-1 in insulin-resistant human skeletal muscle: potential role in insulin resistance. Diabetes. 2003;52:935-41.

56. Yu H, Littlewood T, Bennett M. Akt isoforms in vascular disease. Vascul Pharmacol. 2015;71:57-64.

57. Ding L, Biswas S, Morton RE, Smith JD, Hay N, Byzova TV, Febbraio M, Podrez EA. Akt3 deficiency in macrophages promotes foam cell formation and atherosclerosis in mice. Cell Metab. 2012;15:861-72.

58. Manning BD, Cantley LC. AKT/PKB signaling: navigating downstream. Cell. 2007;129:1261-74.

59. Boyle JJ, Johns M, Kampfer T, Nguyen AT, Game L, Schaer DJ, Mason JC, Haskard DO. Activating transcription factor 1 directs Mhem atheroprotective macrophages through coordinated iron handling and foam cell protection. Circ Res. 2012;110:20-33.

60. Wan X, Huo Y, Johns M, Piper E, Mason JC, Carling D, Haskard DO, Boyle JJ. 5'-AMP-activated protein kinase-activating transcription factor 1 cascade modulates human monocyte-derived macrophages to atheroprotective functions in response to heme or metformin. Arterioscler Thromb Vasc Biol. 2013;33:2470-80.

61. Yang S, Gao Y, Liu G, Li J, Shi K, Du B, Si D, Yang P. The human ATF1 rs 11169571 polymorphism increases essential hypertension risk through modifying miRNA binding. FEBS Lett. 2015:589:2087-93.

62. Bell JT, Tsai PC, Yang TP, Pidsley R, Nisbet J, Glass D, Mangino M, Zhai G, Zhang $\mathrm{F}$, Valdes $\mathrm{A}$, et al. Epigenome-wide scans identify differentially methylated regions for age and age-related phenotypes in a healthy ageing population. PLoS Genet. 2012;8:e1002629.

63. Ma B, Wilker EH, Willis-Owen SA, Byun HM, Wong KC, Motta V, Baccarelli AA, Schwartz J, Cookson WO, Khabbaz K, et al. Predicting DNA methylation level across human tissues. Nucleic Acids Res. 2014;42:3515-28.

64. Ronn T, Volkov P, Gillberg L, Kokosar M, Perfilyev A, Jacobsen AL, Jorgensen SW, Brons C, Jansson PA, Eriksson KF, et al. Impact of age, BMI and HbA1C levels on the genome-wide DNA methylation and mRNA expression patterns in human adipose tissue and identification of epigenetic biomarkers in blood. Hum Mol Genet. 2015;24:3792-813.

65. Guénard F DY, Hould FS, Lebel S, Tchernof A, Marceau P and Vohl MC. Use of Blood as a Surrogate Model for the Assessment of Visceral Adipose Tissue Methylation Profiles Associated with the Metabolic Syndrome in Men. J Mol Genet Med. 2016;10:1

66. Aslibekyan S, Almeida M, Tintle N. Pathway analysis approaches for rare and common variants: insights from Genetic Analysis Workshop 18. Genet Epidemiol. 2014;38 Suppl 1:S86-91.

67. Health Canada. Eating Well with the Canada's Food Guide. Ottawa: Publications Health Canada: 2011

68. Callaway C, Chumlea W, Bouchard C, Himes J, Lohman T, Martin A, Mitchell C, Mueller W, Roche A, Seefeldt V. Standardization of anthropomeric measurements : The Airlie (VA) Consensus Conference. (TG L, AF R, R M editors). Champaign: Human Kinetics; 1988:39-80. pp. 39-80.

69. McNamara JR, Schaefer EJ. Automated enzymatic standardized lipid analyses for plasma and lipoprotein fractions. Clin Chim Acta. 1987;166:1-8.

70. BURSTEIN M, SAMAILLE J. On a rapid determination of the cholesterol bound to the serum alpha- and beta-lipoproteins. Clin Chim Acta. 1960;5:609.

71. Albers JJ, Warnick GR, Wiebe D, King P, Steiner P, Smith L, Breckenridge C, Chow A, Kuba K, Weidman S, et al. Multi-laboratory comparison of three heparin-Mn2+ precipitation procedures for estimating cholesterol in highdensity lipoprotein. Clin Chem. 1978;24:853-6.

72. Friedewald WT, Levy RI, Fredrickson DS. Estimation of the concentration of low-density lipoprotein cholesterol in plasma, without use of the preparative ultracentrifuge. Clin Chem. 1972;18:499-502.

73. Pirro M, Bergeron J, Dagenais GR, Bernard PM, Cantin B, Despres JP, Lamarche B. Age and duration of follow-up as modulators of the risk for ischemic heart disease associated with high plasma C-reactive protein levels in men. Arch Intern Med. 2001;161:2474-80.

\section{Submit your next manuscript to BioMed Central and we will help you at every step:}

- We accept pre-submission inquiries

- Our selector tool helps you to find the most relevant journal

- We provide round the clock customer support

- Convenient online submission

- Thorough peer review

- Inclusion in PubMed and all major indexing services

- Maximum visibility for your research

Submit your manuscript at www.biomedcentral.com/submit
Biomed Central 